\title{
Wireless Sensor Network Communication Using Electromagnetic Waves at Radio Frequency $433 \mathrm{MHz}$
}

\author{
Xiaoqing. $\mathrm{Yu}^{1,2,3}$ and Zenglin.Zhang ${ }^{1,3 *}$ \\ ${ }^{1}$ Northwest A \& F University, Shaanxi, Yangling, 712100, China \\ ${ }^{2}$ North University of China, Shanxi, Taiyuan, 030051, China \\ ${ }^{3}$ Research Institute of Water-saving Agriculture of Arid Regions of China, \\ Shaanxi, Yangling, 712100, China \\ yuxiaoqing115@gmail.com, zhangzenglin115@gmail.com
}

\begin{abstract}
Wireless sensor network has the advantage of being low-cost, easily deployed and of good mobility. Wireless underground sensor network (WUSN) consists of wireless devices that operate below the ground surface. These devices are buried completely under dense soil, thus electromagnetic wave transmits only through soil medium. In this paper, the application research of the wireless underground sensor network is described. Experimental measurements of the signal strength and error rate are presented at the frequency of $433 \mathrm{MHz}$, which show a good agreement with the theoretical studies. The results show the burial depth of the sensor nodes, the horizontal inter-node distance, and the soil volumetric water content have significant impacts on the signal strength and packet error rate during the electromagnetic wave propagation within a WUSN.
\end{abstract}

Keywords: Wireless sensor networks, electromagnetic wave, communication, transmission, soil

\section{Introduction}

A wireless sensor network consists of sensors used to monitor physical or environmental phenomena such as humidity, temperature, sound, pressure or motion and cooperatively pass the data through the network of sensors to a main location (Lopez etal.,2009;Aqeel etal.,2011;Carle and SimPlot-Ryl,2004;Berman etal.,2004;Xin and Mehmet,2010;Sheth, Tejaswi, and Mehta,2005). Wireless underground sensor network is very different from that of traditional wireless sensor network, which electromagnetic wave propagates in soil medium between sensor nodes and the propagation characteristics are decided by soil properties (Akyildiz and Stuntebeck 2006). When a signal propagates within a medium, it may be reflected, diffracted, and scattered (Akyildiz, Sun, and Vuran, 2009; Sun and Akyildiz, 2008). Each effect occurs to a different extent in various media, depending on factors such as wavelength and intensity of the wave, thickness and physical composition of the medium.

Wireless underground sensor network is that the WUSN sensor equipment with wireless transceiver module buried completely in certain soil depth, sensor module percepts the data and send data through the wireless mode. Many sensor nodes consist of sensor network in the soil and complete automatically the entire process of data perception, collection (Yu etal.,2013;Akyildiz etal.,2002; Green, Nadimi, and Blanes,2009;Kima etal.,2011;Shih etal.,2001). The WUSN has many advantages, such as strong concealment, easy of deployment, timeliness of the data, reliability, large covering range, easy of upgrade, etc (Akyildiz and Stuntebeck,2006). 


\section{Related Work}

Wireless underground sensor networks have been investigated in many contexts recently, but research reports of wireless underground sensor networks in agricultural application are little. The concept of WUSN and the challenges related to the underground wireless channel have been introduced in (Akyildiz and Stuntebeck, 2006).

In (Coen, Henk, and Leon,2009), the near surface wireless underground sensor networks system used for golf course was developed which included acquisition nodes, sink nodes and a gateway node. Each acquisition node consisted of a soil moisture sensor, a controller, a wireless transceiver with a carrier frequency of $868 \mathrm{MHz}$, an antenna, a memory unit and a battery power module. It could connect with several moisture sensors. In (Bogena etal.,2009), wireless signal attenuation of ZigBee wireless transceiver module of the $2.44 \mathrm{GHz}$ frequency was researched by using soil column in different soil types and the water content. In ( $\mathrm{Li}$ and Wen,2008), the propagation situation of electromagnetic waves in the soil, underground channel model, electrical characteristics of soil and deployed solutions of wireless underground sensor networks nodes were described. In (Silva and Vuran,2010), the impact factors of the communication performance were studied for terrestrial nodes and underground nodes, including antenna bandwidth of WSN nodes at $433 \mathrm{MHz}$ frequency, the buried depth of nodes in the soil and water content of the soil.

In summary, wireless underground sensor network is a relatively new area which still needs more studies to understand the propagation performance of RF signals. This paper reports the progress on a development of wireless underground sensor network for soil property evaluation and the field experiments to identify significant impact factors on underground RF propagation.

\section{Materials and Methods}

Measurement Principles:

The signal propagation in soil depends on the path loss in soil. Received power as a function of transmitted signal, path loss and antenna gain at the receiver end is given from Friis equation as shown in Equation (1) (Stuber, 2001)

$$
P_{r}(d B m)=P_{t}(d B m)+G_{t}(d B)+G_{r}(d B)-L_{0}(d B)
$$

Where $P_{t}$ is the transmitter power, $G_{t}$ and $G r$ are the gains of the transmitter and receiver antenna, $\mathrm{L}_{0}$ is the path loss of electromagnetic wave propagation in free space. The path loss is shown in Equation (2).

$$
L_{0}(d B)=32.4+20 \log (d)+20 \log (f)
$$

Where $d$ is the distance between transmitting and receiving nodes, measured in meters. $\mathrm{f}$ is node operating frequency, the unit is $\mathrm{MHz}$. Electromagnetic wave propagate in the soil, a correction factor should be increased in Friis equation to express influence of the soil medium on electromagnetic wave propagation loss. As a result, the receiving node as the received signal energy is expressed in Equation (3).

$$
P_{r}(d B m)=P_{t}(d B m)+G_{t}(d B)+G_{r}(d B)-L_{p}(d B)
$$

Where, $\mathrm{L}_{\mathrm{p}}=\mathrm{L}_{0}+\mathrm{L}_{\mathrm{s}}, \mathrm{L}_{\mathrm{s}}$ is extra path loss caused by soil when electromagnetic wave propagate in soil medium. Compared with the air, electromagnetic wave propagation rate will decrease according to the operating frequency, and different band can cause the signal distortion. $\mathrm{S}_{0}$ the extra path loss $\mathrm{L}_{\mathrm{s}}$ caused by soil can be expressed in Equation (4):

$$
L_{s}(d B)=L_{s 1}(d B)+L_{\alpha}(d B)
$$

where, $L_{s 1}$ is attenuation loss due to the difference of the wavelength $\lambda_{1}$ of electromagnetic wave signal in soil, compared to the wavelength $\lambda_{0}$ in free space, and $\mathrm{L}_{\alpha}$ 
is the transmission loss caused by attenuation with attenuation constant $\alpha$, $\mathrm{L}_{\mathrm{s} 1}=20 \log \left(\lambda_{0} / \lambda_{1}\right)$ 。

Considering that the wavelength in the soil propagation is $\lambda_{1}=2 \pi / \beta$, in the free space is $\lambda_{0}=\mathrm{c} / \mathrm{f}$, where $\beta$ is the phase shifting constant, $\mathrm{c}=3 \times 10^{8} \mathrm{~m} / \mathrm{s}, \quad \mathrm{f}$ is the operating frequency, the $\mathrm{L}_{\mathrm{s} 1}$ and $\mathrm{L}_{\alpha}$ can be represented as follows:

$$
\begin{gathered}
L_{s 1}(d B)=154-20 \log (f)+20 \log (\beta) \\
L_{\alpha}(d B)=8.69 \alpha d
\end{gathered}
$$

Given that the path loss in free space is $L_{0}=20 \log \left(4 \pi d / \lambda_{0}\right)$, the path $\operatorname{loss} L_{p}$ of an electromagnetic wave in soil is in Equation (7):

$$
L_{p}(d B)=6.4+20 \log (d)+20 \log (\beta)+8.69 \alpha d
$$

where the distance $d$ is given in meters, the attenuation constant $\alpha$ is in $1 / \mathrm{m}$ and the phase shifting constant $\beta$ is in radian $/ m$. Note that the path loss $L_{p}$ depends on the attenuation constant $\alpha$ and the phase shifting constant $\beta$. The values of these parameters depend on the dielectric properties of soil.

The Peplinski principle governs the value of the complex propagation constant of the electromagnetic wave in soil, which is given as $\gamma=\alpha+\mathrm{j} \beta$ with

$$
\alpha=\omega \sqrt{\frac{\mu \varepsilon^{\prime}}{2}\left[\sqrt{1+\left(\frac{\varepsilon^{\prime \prime}}{\varepsilon^{\prime}}\right)^{2}}-1\right]}
$$

$$
\beta=\omega \sqrt{\frac{\mu \varepsilon^{\prime}}{2}\left[\sqrt{1+\left(\frac{\varepsilon^{\prime \prime}}{\varepsilon^{\prime}}\right)^{2}}+1\right]}
$$

Where $\omega=2 \pi \mathrm{f}$ is the angular frequency, is the magnetic permeability, and $\varepsilon^{\prime}$ and $\varepsilon^{\prime \prime}$ are the real and imaginary parts of the dielectric constant, respectively. Consequently, the path loss in soil is dependent on the complex propagation constant.

Experiment Setup:

The experiment tests were carried out in the laboratory of the Research Institute of Water-saving Agriculture of Arid Regions of China in the Northwest Agriculture and Forestry University. During the experiment, soil medium was assumed as a homogeneous medium, and the surrounding temperature was kept at a range of $20-24{ }^{\circ} \mathrm{C}$ throughout the experiment. Using the pipette method and the Stokes' law (Li and Qi,2001), the soil medium had a clay content of $11.32 \%$, the silt content of $61.26 \%$, and the sand content of $27.42 \%$. The basic physical property index of the soil sample is shown in table 1.

Table 1. The Basic Physical Property Index of the Soil Sample

\begin{tabular}{cccc}
\hline \multirow{2}{*}{ Soil type } & \multicolumn{3}{c}{ Particle-sized fractions $(\%)$} \\
\cline { 2 - 4 } & $\begin{array}{c}\text { Sand } \\
(2 \sim 0.02 \mathrm{~mm})\end{array}$ & $\begin{array}{c}\text { Silt } \\
(0.02 \sim 0.002 \mathrm{~mm})\end{array}$ & $\begin{array}{c}\text { Clay } \\
(<0.002 \mathrm{~mm})\end{array}$ \\
\hline Silty loam & 27.42 & 61.26 & 11.32 \\
\hline
\end{tabular}

In the wireless underground sensor network communication, the sensor nodes are deployed mainly in the underground soil, but it still need to communicate with the ground node to implement the data collection, management and relay, etc. Therefore, there are three different kinds of WUSN communication mode based on the transmitting node and the receiving node are deployed on the aboveground or underground in the soil, the aboveground-underground communication, underground-aboveground and undergroundunderground communication. The diagram of communication structure is shown in Figure 1. 


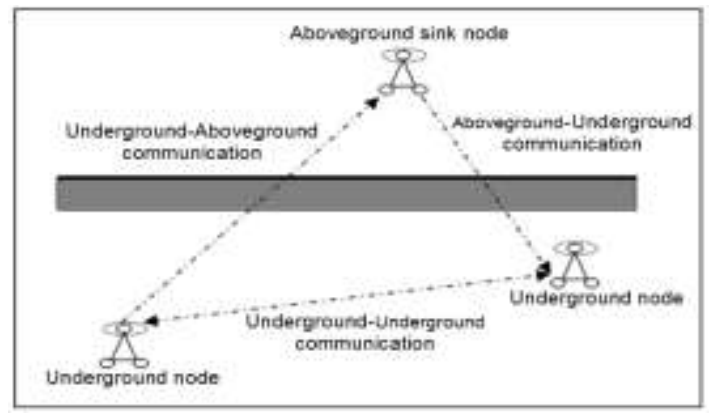

Figure 1. Structure of Communication in WUSN

\section{Results and Discussion}

The aboveground-underground communication:

The aboveground node is deployed on the ground surface that is perpendicular to the underground node, the change of the underground node burial depth and soil moisture content has much effect on the wireless underground sensor network transmission in the WUSN aboveground-underground communication. When the underground node burial depth and soil moisture content change at the same time, received signal strength changes in the range of $-53 \mathrm{dBm}$ to $-110 \mathrm{dBm}$, is shown in Figure 2.

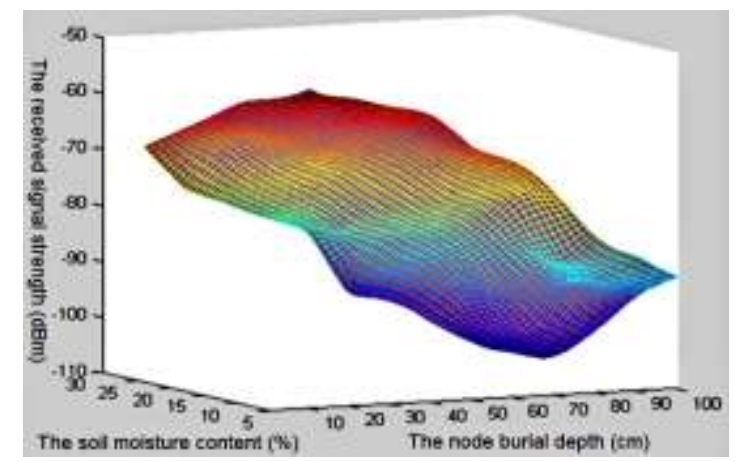

Figure 2. Effects of Received Signal Strength in Aboveground-Underground Communication

It can be included in Figure 2, that the deeper the underground node burial depth, the higher the soil moisture content, received signal strength in the communication is relatively weaker. When soil moisture content is below $20 \%$ and node burial depth changes from $10 \mathrm{~cm}$ to $100 \mathrm{~cm}$, WUSN aboveground-underground communication is feasible, and received signal strength is higher than $-105 \mathrm{dBm}$. When soil moisture content increased to more than $20 \%$, the node burial depth changes in the range of $10 \mathrm{~cm}$ to $90 \mathrm{~cm}$, the received signal strength is greater than $-100 \mathrm{dBm}$, wireless underground sensor networks aboveground-underground communication is good. The analysis is carried on through Matlab, relationship is established in Equation (10).

$$
\begin{gathered}
R_{s s}=-43.5622-0.2722 N_{d}-0.7695 S_{v}-0.0016 N_{d}^{2}-0.0011 N_{d} S_{v}-0.0029 S_{v}{ }^{2} \\
R^{2}=0.9870
\end{gathered}
$$

Where $\mathrm{R}_{\mathrm{ss}}$ is the received signal strength, $\mathrm{dBm} ; \mathrm{N}_{\mathrm{d}}$ is the underground node burial depth, $\mathrm{cm} ; \mathrm{S}_{\mathrm{v}}$ is the soil moisture content, \%。 It can be seen from the Equation (10), the changes of the node burial depth $\mathrm{N}_{\mathrm{d}}$ and soil moisture content $\mathrm{S}_{\mathrm{v}}$ have binary quadratic relationship on the received signal strength $R_{s s}$ in the aboveground-underground communication, goodness-of-fit $\mathrm{R}^{2}$ is higher. 
When the aboveground node is deployed on the ground surface that is perpendicular to the underground node, the change of the underground node burial depth and soil moisture content has much effect on the bit error rate in the WUSN aboveground-underground communication. The bit error rate is shown in Figure 3.

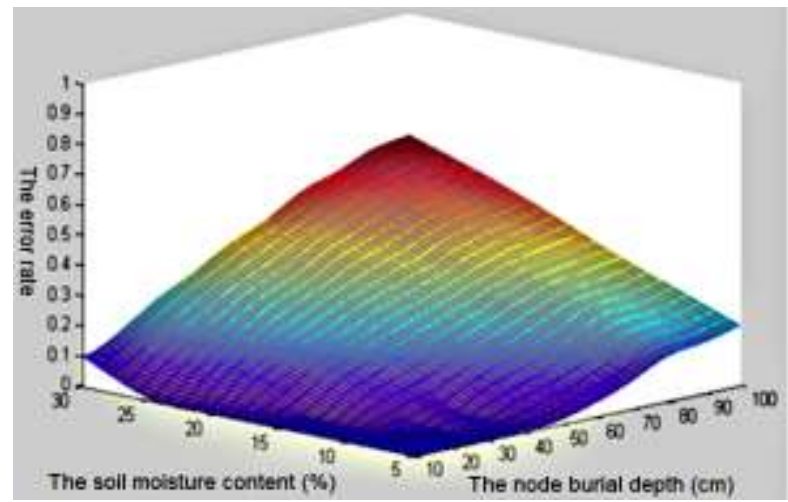

\section{Figure 3. Effects of Packet Error Rate in Aboveground-Underground Communication}

From Figure 3, we can conclude that the deeper the underground node burial depth, the higher the soil moisture content, bit error rate in the communication is relatively weaker. Bit error rate increases with the increase of node burial depth and soil moisture content increase, and the maximum is less than $60 \%$. The analysis is carried on through Matlab, relationship is established in Equation (11).

$$
\begin{gathered}
E_{r}=-0.1353-0.0032 N_{d}-0.0054 S_{v}-0.0001 N_{d} S_{v} \\
R^{2}=0.9821
\end{gathered}
$$

Where $E_{r}$ is the bit error rate; $N_{d}$ is the underground node burial depth, $\mathrm{cm} ; \mathrm{S}_{\mathrm{v}}$ is the soil moisture content, \% 。 It can be seen from the Equation (11), the changes of the node burial depth $\mathrm{N}_{\mathrm{d}}$ and soil moisture content $\mathrm{S}_{\mathrm{v}}$ have binary quadratic relationship on the error rate $E_{r}$ in the aboveground-underground communication, goodness-of-fit $R^{2}$ is higher.

The underground-aboveground communication:

In the wireless underground sensor network communication, undergroundaboveground communication is one of the most important communications. In the process of underground-aboveground communication test, wireless underground sensor network node RF frequency is $433 \mathrm{MHz}$, the aboveground node is deployed on the ground surface that is perpendicular to the underground node, and the change of the received signal strength and the bit error rate are analyzed. The effect of Wireless underground sensor network underground node burial depth and soil moisture content on the received signal strength is shown in Figure 4. 


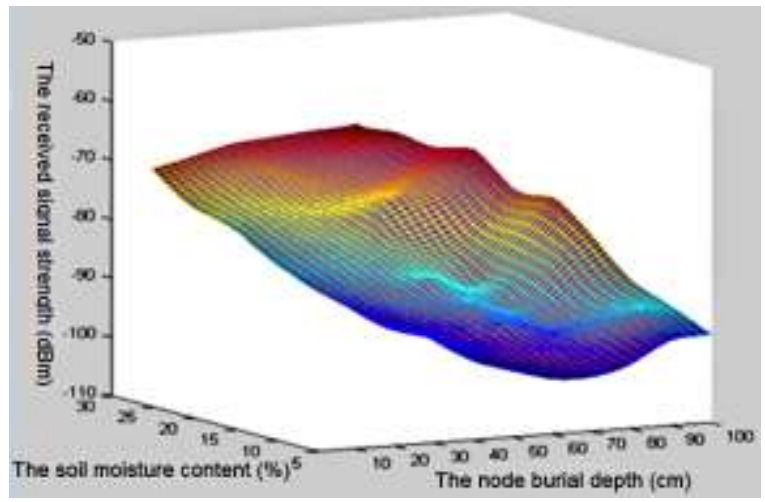

\section{Figure 4. Effects of Received Signal Strength in Underground-Aboveground Communication}

It can be included in Figure 4, that the received signal strength changes from $-55 \mathrm{dBm}$ to $-110 \mathrm{dBm}$ in the WUSN underground-aboveground communication when the aboveground node is deployed on the ground surface that is perpendicular to the underground node. When soil moisture content is below $20 \%$, the received signal strength is higher than $-100 \mathrm{dBm}$. When soil moisture content increased to more than $20 \%$, the received signal strength achieves the minimum $-110 \mathrm{dBm}$ in the maximum underground node burial depth. Under the same soil moisture, the received signal strength decreases by about $2 \mathrm{dBm}$ to $5 \mathrm{dBm}$ in the WUSN underground-aboveground communication compared with the aboveground-underground communication in the same condition. The analysis is carried on through Matlab, relationship is established in Equation (12).

$$
\begin{gathered}
R_{s s}=-38.1378-0.5136 N_{d}-1.4867 S_{v}-0.0003 N_{d}^{2}-0.0010 N_{d} S_{v}-0.0161 S_{v}{ }^{2} \\
R^{2}=0.9869
\end{gathered}
$$

It can be seen from the Equation (12), the changes of the node burial depth $\mathrm{N}_{\mathrm{d}}$ and soil moisture content $S_{\mathrm{v}}$ have binary quadratic relationship on the received signal strength $R_{\mathrm{ss}}$ in the underground-aboveground communication, goodness-of-fit $\mathrm{R}^{2}$ is higher.

Figure 5 reflects the change of bit error rate in the wireless underground sensor network underground-aboveground communication under the underground node burial depth and soil moisture content change circumstances.

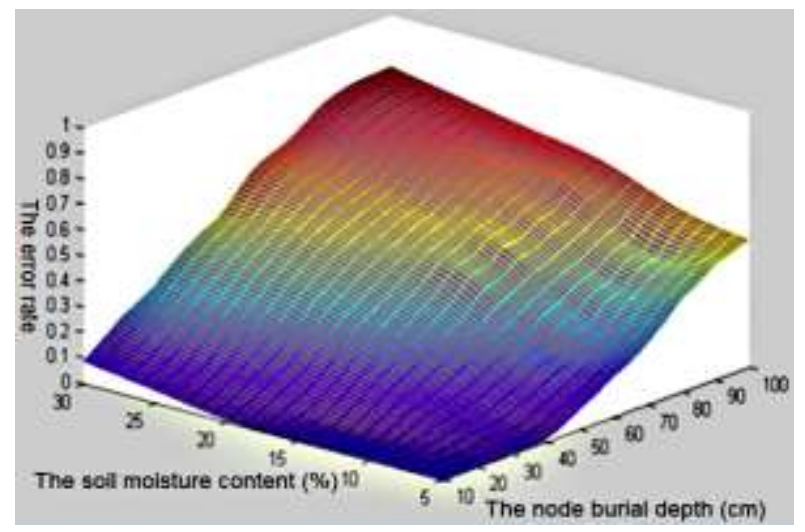

\section{Figure 5. Effects of Packet Error Rate in Underground-Aboveground Communication}

From Figure 5, we can conclude that underground node burial depth and soil moisture content change within the biggest scope, the bit error rate is under $80 \%$ in the WUSN underground-aboveground communication, the communication is good. The bit error rate is less than $70 \%$ when node burial depth is less than $80 \mathrm{~cm}$, soil moisture content changes 
within the scope of $5 \%$ to $30 \%$. With the increases of node burial depth, the bit error rate increases, but the maximum error rate is less than $80 \%$. In addition, when the soil moisture content is less than $20 \%$, the node burial depth changes from $10 \mathrm{~cm}$ to $100 \mathrm{~cm}$, bit error rate is still less than $70 \%$. With the increases of soil moisture content, the bit error rate also increases slightly. Compared with the aboveground-underground communication in the same condition, the bit error rate is greater in the WUSN underground-aboveground communication and increases by about $20 \%$ to $30 \%$. The analysis is carried on through Matlab, relationship is established in Equation (13).

$$
\begin{gathered}
E_{r}=-0.2289-0.0060 N_{d}-0.0010 S_{v}-0.0001 N_{d} S_{v}-0.0001 S_{v}{ }^{2} \\
R^{2}=0.9703
\end{gathered}
$$

It can be seen from the Equation (13), the changes of the node burial depth $\mathrm{N}_{\mathrm{d}}$ and soil moisture content $S_{v}$ have binary quadratic relationship on the error rate $E_{r}$ in the underground-aboveground communication, goodness-of-fit $\mathrm{R}^{2}$ is higher.

The underground-underground communication:

To investigate the effects of burial depth and water moisture content on the received signal strength and bit error rate, the horizontal inter-node distance between the sender and the receiver is fixed $50 \mathrm{~cm}$. In the communication between underground nodes, the sender node is fixed depth $40 \mathrm{~cm}$, the receiver node varies from $10 \mathrm{~cm}$ to $100 \mathrm{~cm}$. Moreover, the range of the soil moisture content is 5\% to 30\%. In Figure 6 and Figure 7, the received signal strength and bit error rate values are shown, respectively.

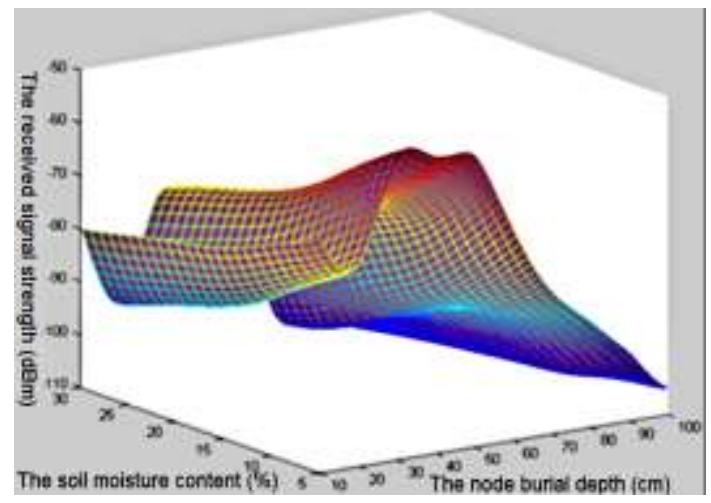

Figure 6. Tests for the Received Signal Strength. The Underground Node Is Varied From $10 \mathrm{~cm}$ to $100 \mathrm{~cm}$

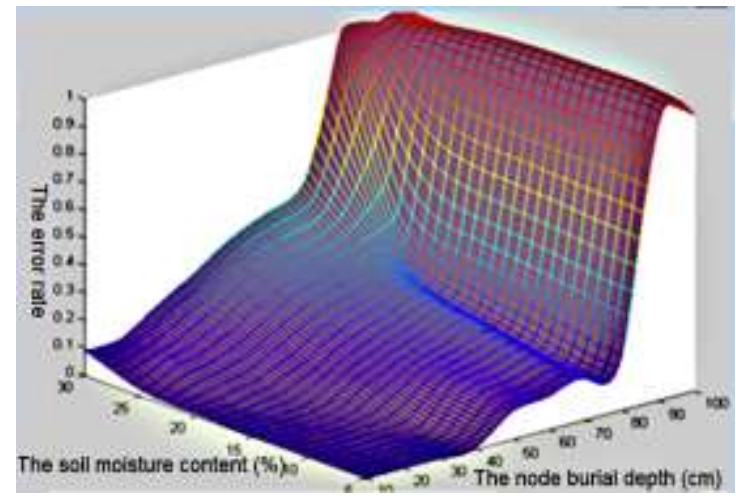

Figure 7. Tests for Bit Error Rates. The Underground Received Node Is Varied From $10 \mathrm{~cm}$ to $100 \mathrm{~cm}$

It can be observed in Figure 6, that received signal strength gradually reduced with the increase of the receiver node depth when WUSN sender node buried depth keeps in depth $40 \mathrm{~cm}$. When receiver node burial depth changes from $30 \mathrm{~cm}$ to $50 \mathrm{~cm}$, 
received signal strength reaches maximum. Received signal strength gradually increases when receiver node buried depth is less than $30 \mathrm{~cm}$ and change to $10 \mathrm{~cm}$, received signal strength gradually decreases when receiver node buried depth is more than $50 \mathrm{~cm}$.

In addition, we can conclude that soil moisture content is less than $30 \%$, the minimum received signal strength is higher than $-110 \mathrm{dBm}$. The received signal strength reaches the minimum when the soil moisture content is $30 \%$. With the increase of soil volumetric water content interval $5 \%$, received signal strength reduces $2 \mathrm{dBm}$ to $10 \mathrm{dBm}$ when the receiver node burial depth below $80 \mathrm{~cm}$. The decrease extent of the received signal strength becomes small when receiver node buried depth is higher than $80 \mathrm{~cm}$, average 1 $\mathrm{dBm}$. The effect of the receiver node burial depth and the soil moisture content is analyzed through Matlab, relationship is established in Equation (14).

$$
\begin{gathered}
R_{s s}=-53.8017+0.2083 N_{d}-1.8269 S_{v}-0.0065 N_{d}^{2}+0.0048 N_{d} S_{v}+0.0231 S_{v}{ }^{2} \\
R^{2}=0.814
\end{gathered}
$$

It can be seen from the Equation (14), the changes of the receiver node burial depth $\mathrm{N}_{\mathrm{d}}$ and soil moisture content $S_{\mathrm{v}}$ have binary quadratic relationship on the received signal strength $R_{\mathrm{ss}}$ in the underground-underground communication, goodness-of-fit $\mathrm{R}^{2}$ is higher.

It can be included in Figure 7, that WUSN node electromagnetic wave communication produces the error rate in the underground soil that increases with the increase of soil moisture content. When the soil moisture content changes in the rang of 5\% to $30 \%$ and receiver node burial depth $10 \mathrm{~cm}$ to $70 \mathrm{~cm}$, the highest error rate is no more than $50 \%$. The error rate increases with the increase of soil moisture content, when the receiver node burial depth $80 \mathrm{~cm}$ and soil moisture content changes from $5 \%$ to $25 \%$, the maximum error rate is about $40 \%$. The effect of the receiver node burial depth and the soil moisture content is analyzed through Matlab, relationship is established in Equation 15.

$$
\begin{gathered}
E_{r}=0.1313-0.0098 N_{d}-0.0101 S_{v}+0.0002 N_{d}{ }^{2}+0.0005 S_{v}{ }^{2} \\
R^{2}=0.8845
\end{gathered}
$$

It can be seen from the Equation (15), the changes of the node burial depth $\mathrm{N}_{\mathrm{d}}$ and soil moisture content $S_{v}$ have binary quadratic relationship on the error rate $E_{r}$ in the underground-underground communication, goodness-of-fit $\mathrm{R}^{2}$ is higher.

In the test of underground-underground communication, the sensor node RF frequency uses $433 \mathrm{MHz}$, sender and receiver nodes burial depth are fixed $40 \mathrm{~cm}$, soil moisture content changes in the range of $5 \%$ to $30 \%$, the change range of horizontal inter-node distance is $10 \mathrm{~cm}$ to $100 \mathrm{~cm}$. The effects of horizontal inter-nodes distance and soil moisture content on received signal strength and the error rate are measured, as shown in Figure 8 and Figure 9.

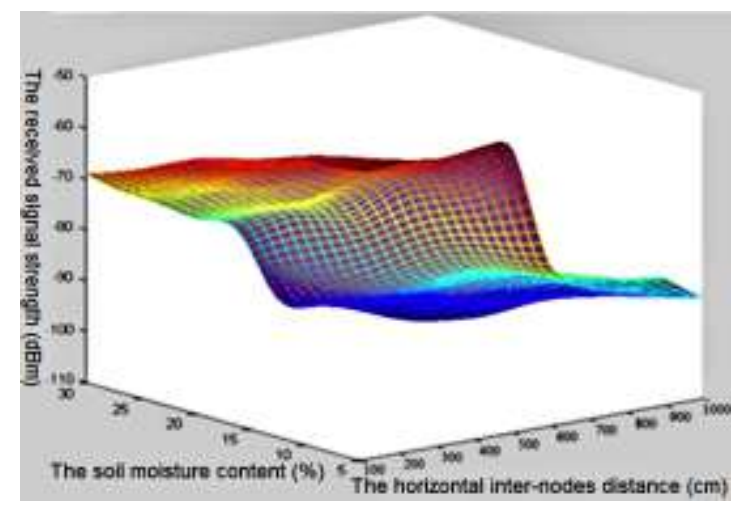

Figure 8. Tests for the Received Signal Strength. The Horizontal Inter-Nodes Distance Varies From $10 \mathrm{~cm}$ to $100 \mathrm{~cm}$ 


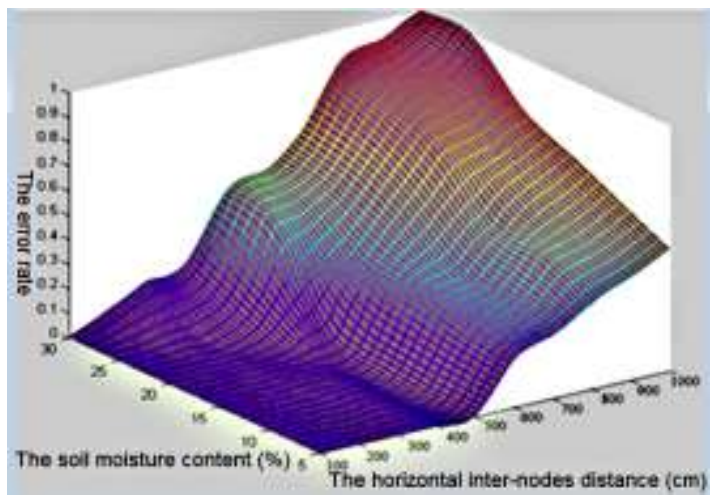

\section{Figure 9. Tests for Packet Error Rates. The Horizontal Inter-Nodes Distance Is Varied From $10 \mathrm{~cm}$ to $100 \mathrm{~cm}$}

It can be showed in Figure 8, when the horizontal inter-nodes distance changes in range of $10 \mathrm{~cm}$ to $100 \mathrm{~cm}$ and soil moisture content is less than $25 \%$, the minimum received signal strength is not less than $-100 \mathrm{dBm}$. The received signal strength gradually decreases with the increase of the horizontal inter-nodes distance when the soil moisture content is $25 \%$, the minimum value is about $-105 \mathrm{dBm}$. When soil moisture content increases continually to $30 \%$, the received signal strength reduces and reaches minimum, about $-110 \mathrm{dBm}$. The effect of the horizontal inter-nodes distance and the soil moisture content is analyzed through Matlab, relationship is established in Equation (16).

$$
\begin{gathered}
R_{s s}=-37.8433-0.0502 H_{s}-0.5746 S_{v}-0.0103 S_{v}{ }^{2} \\
R^{2}=0.9449
\end{gathered}
$$

It can be seen from the Equation (16), the changes of he horizontal inter-nodes distance $\mathrm{H}_{\mathrm{s}}$ and soil moisture content $\mathrm{S}_{\mathrm{v}}$ have binary quadratic relationship on the received signal strength $R_{s s}$ in the underground-underground communication, goodness-of-fit $R^{2}$ is higher.

We can include from Figure 9, the error rate increases gradually when the soil moisture content is less than $25 \%$ and the horizontal inter-nodes distance changes in the range of 10 $\mathrm{cm}$ to $100 \mathrm{~cm}$. At the maximum horizontal inter-nodes distance, the error rate increase extent is almost equal to $10 \%$, but the highest error rate is no more than $80 \%$. When soil moisture content is more than $25 \%$, the error rate reaches $100 \%$ in the maximum horizontal inter-nodes distance. The effect of the horizontal inter-nodes distance and the soil moisture content is analyzed through Matlab, relationship is established in Equation (17).

$$
\begin{gathered}
E_{r}=0.0346-0.0003 H_{s}-0.0113 S_{v}+0.0003 S_{v}{ }^{2} \\
R^{2}=0.9630
\end{gathered}
$$

It can be seen from the Equation (17), the changes of the horizontal inter-nodes distance $H_{s}$ and soil moisture content $S_{v}$ have binary quadratic relationship on the error rate $E_{r}$ in the underground-underground communication, goodness-of-fit $R^{2}$ is higher.

In a word, the node burial depth, the horizontal inter-nodes distance and the soil moisture content have a great impact on the received signal strength and bit error rate of the aboveground-underground, underground-aboveground and underground-underground communication. 


\section{Conclusions}

In this work, we propose the characteristics of aboveground-underground, underground-aboveground and underground-underground communication in the WUSN. The experiment design and results were presented in this paper. The experiment results revealed the feasibility of RF wave transmission in the soil medium for wireless underground sensor networks and showed the effect of some influence factors on underground communication. From above experiments, we could conclude that:

(1) In the WUSN aboveground-underground communication, the received signal strength changes through binary quadratic relationship in the range of $-53 \mathrm{dBm}$ to -110 $\mathrm{dBm}$ when node burial depth and soil moisture content change, and the error rate is less than $60 \%$.

(2) The received signal strength changes through binary quadratic relationship in the range of $-55 \mathrm{dBm}$ to $-110 \mathrm{dBm}$ when node burial depth and soil moisture content change in the underground-aboveground communication, and the error rate is not more than $80 \%$. Compared with the aboveground-underground communication, the received signal strength decreases by about $2 \mathrm{dBm}$ to $5 \mathrm{dBm}$ in the underground-aboveground communication.

(3) In the underground-underground communication, the received signal strength decreases with the increase of soil moisture content when node burial depth and soil moisture content change, and has the relationship of binary quadratic with node burial depth and soil moisture content. When the soil moisture content is $20 \%, 25 \%, 30 \%$ and the horizontal inter-nodes distance is $1000 \mathrm{~cm}, 1000 \mathrm{~cm}, 600 \mathrm{~cm}$, respectively, the received signal strength achieves $-100 \mathrm{dBm}$. The received signal strength and the error rate change through binary quadratic relationship with the change of the horizontal internodes distance and soil moisture content.

\section{Acknowledgements}

The authors wish to thank the National Engineering Research Center for Water-Saving Irrigation, which partially supported this research through the " National Natural Science Funds of China (51509207)" . The authors are also grateful to the anonymous reviewers for their valuable feedback.

\section{References}

[1] J. A. Lopez Riquelme, F. Soto and J. Suardiaz, "Wireless Sensor Networks for precision horticulture in Southern Spain", Computing \& Electronics in Agriculture Journal, vol. 68, no. 1, (2009), pp.25-35.

[2] R. Aqeel, Z. A. Abu, I. Noman and A.S.Zubair, "A review of wireless sensors and networks applications in agriculture", Computer Standards \& Interfaces, vol. 36, no. 2, (2011), pp.263-270.

[3] J. Carle and D. SimPlot-Ryl, "Energy Efficient Area Monitoring by Sensor Networks", IEEE Computer Magazine, vol. 37, no. 2, (2004), pp.40-46.

[4] P. Berman, G. Calinescu, C. Shah and A. Zelikovsky, "Power efficient Monitoring Management in Sensor Networks", Proceedings of IEEE Wireless Communication and Networking Conference, (2004), pp.2329-2334.

[5] D. Xin and C. Mehmet, "Spatio-temporal Soil Moisture Measurement with Wireless Underground Sensor Networks", Ad hoc Networking Workshop,(2010), pp.1-8.

[6] A. Sheth, K. Tejaswi and P. Mehta, "Senslide: A Sensor Network Based Landslide Prediction System", The 3rd International Conference on Embedded Networked Sensor Systems, (2005), pp. 280281.

[7] I. F. Akyildiz and E. P. Stuntebeck, "Wireless underground sensor networks: Research challenges", Ad Hoc Networks, vol. 6, no. 4, (2006), pp.669-686.

[8] I. F. Akyildiz, Z. Sun and M. C. Vuran, "Signal propagation techniques for wireless underground communication networks", Physical Communication, vol. 3, no. 2, (2009), pp.167-183.

[9] Z. Sun and I. F. Akyildiz, "Channel Modeling of Wireless Networks in Tunnels", Global Telecommunications Conference 2008, IEEE Globecom 2008, (2008), pp.1-5. 
[10] X. Q. Yu, P. T. Wu, W. T. Han and Z. L. Zhang, “A survey on wireless sensor networks infrastructure for agriculture", Computer Standards \& Interfaces, vol. 35, no. 1, (2013), pp.59-64.

[11] I. F. Akyildiz, W. Su, Y. Sankarasubramaniam and E. Cayirci, "Wireless Sensor Networks: a survey", Computer Networks, vol. 4, no. 38, (2002), pp.393-422.

[12] O. Green, E.S. Nadimi and V. Blanes, "Monitoring and modeling temperature variations inside silage stack using novel wireless sensor networks", Computers and Electronics in Agriculture, vol. 69, no. 2, (2009), pp.149-157.

[13] Y. Kima, Y. M. Yangb and W. S. Kanga, "On the Design of Beacon based Wireless Sensor Network for Agricultural Emergency Monitoring Systems”, Computer Standards \& Interfaces, vol. 5, no. 4, (2011), pp.32-39.

[14] E. Shih, S. Cho, N. Ickes, R. Min, A. Sinha, A. Wang and A. Chandrakasan, "Physical Layer Driven Protocol and Algorithm Design for Energy-Efficient Wireless Sensor Networks", Proceedings of 2001 ACM MOBICOM, (2001), pp. 272-287.

[15] J. R. Coen, K. Henk and K. Leon, "A new wireless underground network system for continuous monitoring of soil water contents", Water resources research, vol. 45, no. 36, (2009), pp.36-44.

[16] H. R. Bogena, J. A. Huismana, H. Meierb, U. Rosenbauma and A. Weuthena, "Hybrid wireless underground sensor networks: Quantification of signal attenuation in soil”, Vadose Zone Journal, vol. 8, no. 3, (2009), pp.755-761.

[17] L. Li and X. M. Wen, "Energy Efficient Optimization of Clustering Algorithm in Wireless Sensor Network", Journal of electronics \& information technology, vol. 30, no. 4, (2008), pp.966-969

[18] A. R. Silva and M. C. Vuran, "Communication with aboveground devices in wireless underground sensor networks: An empirical study", 2010 IEEE International Conference Proceedings, 23-27 May 2010, (2010), pp.1-6.

[19] G. Stuber, "Principles of Mobile Communication”, Klumer Academic Publishers, (2001).

[20] Y. H. Li and J. X. Qi, "Design of Detecting System for Powder Particle Size", Instrument Technique and Sensor, vol. 8, (2011), pp.98-100. 
International Journal of Hybrid Information Technology

Vol. 10, No.1 (2017) 